\title{
Antagonistic activities of bioagent fungi Trichoderma harzianum and Pleurotus ostreatus against three species of Fusarium in cucumber plants
}

\author{
Nasir A. Hussein ${ }^{\mathrm{a}}$, Haider J. K. Al-Janabi ${ }^{\mathrm{b}}$, Fatimah R. Al-Mashhadyc, Jawad K. Abood Al-Janabi, ${ }^{\mathrm{a}, \mathrm{c}^{*}}$, \\ Ali R. Shakir Al-Shujairia \\ ${ }^{a}$ College of Science, University of Babylon, Babylon Province, Iraq \\ ${ }^{b}$ Al-Mustaqbal University College, Babylon Province, Iraq \\ 'Hilla University College, Babylon Province, Iraq
}

Received 13th July 2021 / Accepted 8th October 2021

\begin{abstract}
Many bioagent fungi have promising potential as eco-friendly alternatives to fungicides, with considerable antagonistic activity against various phytopathogenic fungi. The present study aimed to investigate the antagonistic activity of Trichoderma harzianum and Pleurotus ostreatus isolates against Fusarium spp., the causative agents of wilt disease in cucumber plants, through a dual plate assay of volatile and nonvolatile compounds from these bioagent fungi. The results showed significant $(\mathrm{P}<0.05)$ antagonistic activities of $T$. harzianum against the growth of F. solani AJA2 (62.3\%), followed by F. oxysporum AJA (55.2\%), F. incarnatum AJA (53.2\%), and F. solani AJA1 (50.8\%). The effectiveness of P. ostreatus against the four Fusarium species was notably less than that of T. harzianum. In contrast, in the dual culture assay, the bioagent fungal filtrate exhibited inhibitory effects on the growth of all pathogens at $25 \%$ concentration. The highest inhibition rate $(85 \%)$ was shown by T. harzianum against F. incarnatum. The percent of inhibition caused by $P$. ostreatus was substantially lower than that caused by T. harzianum, which reached 35\% in F. incarnatum followed by other pathogens. The volatile compounds of T. harzianum led to a high percentage of inhibition of all the three Fusarium species, while the highest percentage of inhibition due to the compounds of $P$. ostreatus was observed only for F. solani AJA1 (41.5\%). From these results, we concluded that despite the diverse inhibitory effects of both bioagent fungi against Fusarium species, they exhibited successful antagonistic activity and the ability to compete against these species.
\end{abstract}

Keywords: Fusarium species, Trichoderma haræianum, Pleurotus ostreatus, antagonistic activities.

\section{INTRODUCTION}

Endophytic fungi have been shown to possess several advantages in terms of reducing plant diseases, supporting rich biodiversity, serving as a bioresource for bioremediation of organic pollutants and heavy metals, and promoting agricultural sufficiency; thus, making them an important component of sustainable development in community ecology (Mishra \& Sarma, 2018; Chen et al., 2020). Several companies are developing biopesticides and biofertilizers that can be used instead of synthetic chemicals, which could reduce the amount of chemical residues in the environment. Trichoderma is a well-known fungus with a wide distribution. Previous studies on fungal interactions mediated by Trichodermaproduced volatile organic compounds (VOCs) (Oszako et al., 2021; Rajani et al., 2021) have demonstrated that Trichoderma species play a significant role in antagonizing and suppressing the growth of several plant pathogenic fungi.

*Author for correspondence: Jawad K. Abood Al-Janabi, College of Science, University of Babylon and Hilla University College, Babylon Province, Iraq. Email - jka.uobsci.iq@gmail.com 
Moreover, it has been hypothesized that VOCs may play a role in this inhibitory effect of Trichoderma. Understanding the key drivers of VOC production during fungal coculture could be useful for leveraging this knowledge in using endophytes to combat plant pathogenic fungi. A previous study investigated the potential of four Trichoderma species to produce VOCs and examined their inhibitory effects on the growth of Fusarium oxysporum, while another study investigated the synthesis of bioactive VOCs from T. atroviride under a wide range of conditions and assessed their activity against the pathogenic fungus Rhizoctonia (Kottb et al., 2015). The crude extract of Trichoderma contains a wide range of secondary metabolites that may possess antimicrobial capabilities, and it has been used to assess the potential of this fungus in biocontrol and other industrial applications ( $\mathrm{Li}$ et al., 2018). Speckbacher et al. (2020) investigated the antifungal activities of culture filtrates obtained from four Pleurotus spp. in liquid and solid media against a variety of phytopathogenic fungi. The authors concluded that Pleurotus ostreatus culture filtrate had the highest antifungal activity against pathogenic fungi. Marques et al. (2018) demonstrated that Pleurotus salmoneostramineus liquid filtrate shows high antimicrobial activity against Pseudomonas aeruginosa and Candida parapsilosis. Pleurotus cornucopiae mycelia suppressed the growth of Enterococcus faecalis and C. parapsilosis by $5.21 \%$ and $29.19 \%$, respectively. Thus far, chemical fungicides have been used excessively and continuously for crop protection, thereby posing major health risks to humans, animals, and the environment. Therefore, alternative crop protection approaches must be investigated, and one of the potential methods that is both consumer and environmental friendly is the use of biological control agents and their secondary metabolites (Owaid et al., 2015; Owaid et al., 2017). The present study aimed to investigate the antagonistic potential of $T$. harzianum and $P$. ostreatus isolates against Fusarium solani, Fusarium oxysporum, and Fusarium incarnatum, the causative agents of root rot and wilt in cucumber plants, through an in vitro antagonist assay and assessment of the effect of volatile metabolites and fungal filtrate of these bioagent fungi under laboratory conditions.

\section{MATERIALS AND METHODS}

\section{Source of bioagent and pathogenic fungal isolates}

$P$. ostreatus isolate was kindly given and identified by Prof. Dr. Majeed M. Dewan, College of Agriculture, University of Kufa, Iraq. T. harzianum AJA, F. incarnatum AJA, F. solani AJA1, F. solani AJA2, and F. oxysporum AJA were morphologically and molecularly characterized and described in previous studies (Heydari et al., 2010). In this experiment, $P$. ostreatus and T. harzianum AJA were used as bioagent fungi, while the four strains of Fusarium spp. were used as pathogenic fungi.

According to the type of experiment, fungal isolates were recultured on potato dextrose agar (PDA) by taking a $0.5 \mathrm{~cm}$ (diameter) mycelial disc from the edge of the most recent colony of each fungus and placing it on the center of a Petri plate containing sterilized PDA supplemented with chloramphenicol $(0.05 \mathrm{~g} / \mathrm{L})$ at $\mathrm{pH}$ 5.6; the plate was incubated at $25 \pm 1^{\circ} \mathrm{C}$ for 7 days and then stored at $5^{\circ} \mathrm{C}$.

\section{In vitro antagonist assay}

The antagonistic potential of the bioagent fungi against the fungal pathogens (F. incarnatum AJA, F. solani AJA1, F. solani AJA2, and F. oxysporum AJA) was determined using the dual culture method (Khan et al., 2020). A mycelial disc $(5 \mathrm{~mm}$ diameter) was taken from the edge of a 5-day-old culture of each pathogen and placed $1 \mathrm{~cm}$ away from the periphery of the Petri plate $(90 \mathrm{~mm})$, and a disc of the same size for each tested bioagent fungus was placed in the same way but at the opposite end of the pathogen sample. The mycelium plug of each Fusarium strain was inserted in the periphery of the Petri plates in the control treatment. The plates were incubated at $25 \pm 1^{\circ} \mathrm{C}$ in triplicate for each pairing. The average of two crossing lines in the plate's center was used to calculate radial growth (Kuzmanovska et al., 2018; Nasir et al., 2021). These tests were conducted when the pathogen colonized the entire surface of the medium in the control treatment.

The percent growth inhibition (PGI) was determined on the 7 th day of the test by using the following equation (Miyashira et al., 2010): 


$$
P G I=[(C-T) / C \times 100)]
$$

Where PGI=Percent Growth Inhibition (\%); $\mathrm{C}=$ Pathogen Radial Growth (Control); $\mathrm{T}=$ Pathogen Radial Growth (Test).

\section{Preparation of T. harzianum and P. ostreatus extracellular culture filtrate}

The potential of culture filtrates of T. harzianum and $P$. ostreatus isolates against the plant pathogenic fungi $F$. incarnatum AJA, $F$. solani AJA1, F. solani AJA2, and F. oxysporum AJA was evaluated using the method proposed by Fokkema (1978) and Tamur et al. (2018). Both bioagents and the pathogenic fungi were cultivated on PDA in plastic Petri plates. The cultures were incubated for 7 days at $25 \pm 1^{\circ} \mathrm{C}$. $T$. harzianum and $P$. ostreatus isolates were grown in potato dextrose broth (PDB) by inoculating the medium with a $0.5 \mathrm{~mm}$ mycelial disc of a 5 -dayold culture followed by incubation at $25 \pm 1{ }^{\circ} \mathrm{C}$ on a shaker at $140 \mathrm{rpm}$ in dark for 10 days to obtain the liquid phase with nonvolatile metabolites.

After twice filtration through a Whatman No. 1 filter paper, the fungal culture was centrifuged and sterilized using a membrane filter with a pore size of $0.22 \mathrm{~mm}$. The filtrate was kept in the refrigerator in a dark bottle until required for analysis (Ali et al., 2020).

\section{Antagonistic efficacy of extracellular culture filtrates}

The culture filtrates of both bioagent fungi were tested as antifungal agents in this study. For this purpose, 5-mm-diameter agar discs were cut from the cultures of F. incarnatum AJA, F. solani AJA1, F. solani AJA2, and F. oxysporum AJA and placed in the center of each Petri plate containing $(25 \%$ $\mathrm{v} / \mathrm{v}$ ) culture filtrate. A similar procedure was performed in the control treatment by using distilled water instead of culture filtrate. The experiment was replicated thrice. Radial mycelial growth and percentage inhibition of the pathogens were assessed as mentioned in earlier (section: In vitro antagonist assay).

\section{Volatile compound interaction assay}

The effect of volatile compounds produced by $T$. harizianum and P. ostreatus on the growth of the four strains of Fusarium species was investigated using the sandwiched Petri plate method (Fokkema,
1978), with some modifications. On the top surface of T. harzianum and P. ostreatus Petri plates, each Fusarium strain was placed individually. Three layers of parafilm were used to seal the plates, and the plates were then incubated at $25 \pm 1{ }^{\circ} \mathrm{C}$. Each plate of pathogenic fungus was sandwiched between two uninoculated PDA plates (control treatment). The colony diameter and the percentage growth inhibition of Fusarium species were measured as described in section: In vitro antagonist assay.

\section{Statistical analysis}

Statistical data are presented as mean and standard deviation (SD). A one-way analysis of variance (ANOVA) was used to analyze the data, and Duncan's Multiple Range Test was used to assess whether differences in mean values were significant at $\alpha$ level of 0.05 (IBM SPSS Statistics software version 20.0).

\section{RESULTS}

\section{Antagonistic activity of $T$. harzianum and $P$. ostreatus against the growth of Fusarium species}

As shown in Figure 1-G1 (A1 to D1), the growth of F. incarnatum AJA (A1), F. solani AJA1 (B1), F. solani AJA2 (C1), and F. oxysporum AJA (D1) treated with $T$. barzianum was substantially diminished, and $T$. harzianum mycelia mostly covered the Petri plates of all pathogenic fungi after 7 days of incubation (A2 to D2). This antagonistic fungus grew rapidly and had a substantial inhibitory effect on the growth of the four Fusarium strains.

A similar tendency was noted for the treatment of F. incarnatum AJA, F. solani AJA1, F. solani AJA2, and F. oxysporum AJA with $\mathrm{P}$. ostreatus (A3 to D3). The antagonistic fungus covered the entire Petri plate within 7 days of incubation and grew very rapidly. It was able to grow over the mycelial growth of the pathogens with an increase in the incubation period. All pathogens were susceptible to $P$. ostreatus but to a varying degree. Both $P$. ostreatus and T. harzianum covered the entire Petri plate and did not allow the pathogenic fungi to grow as they actively 
colonized the plate inoculated with Fusarium species.

The PGI of pathogenic fungi in a solid culture medium was determined by dual culture testing of the interaction between $T$. harianum and pathogenic fungi mycelia. As shown in Figure 1G2 (S1), the highest antagonistic activities were observed against F. solani AJA2 (62.3\%), followed by F. oxysporum AJA (55.2\%), F. incarnatum AJA (53.2\%), and F. solani AJA1 (50.8\%).

P. ostreatus (Figure 1-S2) showed the highest inhibitory activity against $F$. solani AJA2 (58.4\%), followed by F. oxysporum AJA (50.7\%), F. solani AJA1 (47.2\%), and F. incarnatum AJA (44.6\%). The results showed a significant difference $(\mathrm{P}<0.05)$ in the PGI of the four Fusarium strains under the antagonistic activity of $T$. harzianum and P. ostreatus.

\section{Antagonistic effect of nonvolatile compounds on the growth of Fusarium species}

The results shown in Figure 2-G1 (A2 to D2) revealed that the T. harzianum filtrate completely inhibited the colony growth of all the four Fusarium strains.

Despite using an appropriate filtration procedure to obtain a pure T. harzianum fungal filtrate, many spores passed through the filter, which increased the antagonistic ability of this bioagent fungus to cover the entire plate and prevent pathogen growth. Therefore, a high inhibitory effect on the fungal pathogens was observed due to the direct growth of $T$. harzianum spores from the filtrate, which completely dominated the colony development of Fusarium species. In contrast, the mycelial growth of all the four Fusarium strains covered the entire surface of the untreated Petri plates during the same incubation period. The filtrate of $P$. ostreatus greatly affected the morphological characteristics such as the color, texture, and nature of colonies of all Fusarium species (Figure 2 right side). The filtrate of $P$. ostreatus inhibited the growth of all the four Fusarium strains, and the maximum inhibition was observed for $F$. incarnatum, followed by $F$. oxysporum AJA, F. solani AJA2, and F. solani AJA1. In contrast, the mycelial growth of Fusarium species in control samples covered the entire plate. In the dual culture assay, the fungal filtrate of $T$. harzianum and $P$. ostreatus showed varying inhibitory effects on the growth of all pathogens (Figure 2-G2 (S1 and S2)). The fungal filtrate at $25 \% \quad(\mathrm{v} / \mathrm{v})$ concentration caused substantial changes in the colony morphology of the pathogens, with a significant reduction $(\mathrm{P} \leq 0.05)$ in the growth of the pathogenic fungi. The percentage inhibition varied according to the type of bioagent fungus and the strain of Fusarium species. The highest inhibition was caused by $T$. harzianum (Figure 2-G2 S1) in F. incarnatum AJA (85\%), followed by F. solani AJA1 and F. solani AJA2 (76\%) and F. oxysporum AJA (68\%). In contrast, the percent inhibition caused by $P$. ostreatus (Figure 2-G2S2) was substantially lower than that caused by $T$. harzianum; the percent inhibition was 35\% in F. incarnatum AJA, followed by F. oxysporum AJA (18.3\%), F. solani AJA1 $(13.2 \%)$, and F. solani AJA2 (12.6\%).

\section{Antagonistic effects of volatile compounds on the growth of Fusarium species}

Figure 3-G1 (A2 to D2) shows that the pathogens F. incarnatum AJA, F. solani AJA1, F. solani AJA2, and F. oxysporum AJA not treated with the bioagent fungi grew very rapidly and covered the entire plates in control cultures in 7 days, whereas the growth of these pathogenic fungi that came into contact with volatile compounds released from the bioagent fungi (T. harianum (A2 to D2) or P. ostreatus (A3 to D3)) was restricted with substantial morphological changes.

Despite differences in the inhibitory effect of T. harzianum and P. ostreatus on the growth of the Pathogenic Fusarium strains (Figure 3-G2 (S1 and S2)), the results revealed that both bioagent fungi produce potentially toxic volatiles that significantly affected the radial growth of the pathogenic fungi. The volatile compounds of $T$. harianum (G2:S1) increased the percentage inhibition of all the pathogenic fungi; the highest inhibition was observed for F. incarnatum (50\%), followed by F. oxysporum (35.1\%), F. solani AJA2 $(27.2 \%)$, and F. solani AJA1 (12.5\%). The volatile compounds of $P$. ostreatus (G2:S2) caused the highest inhibition of $F$. solani AJA1 (41.5\%), followed by F. oxysporum (36.2\%), F. solani AJA2 (31.2\%), and F. incarnatum (18.1\%). 


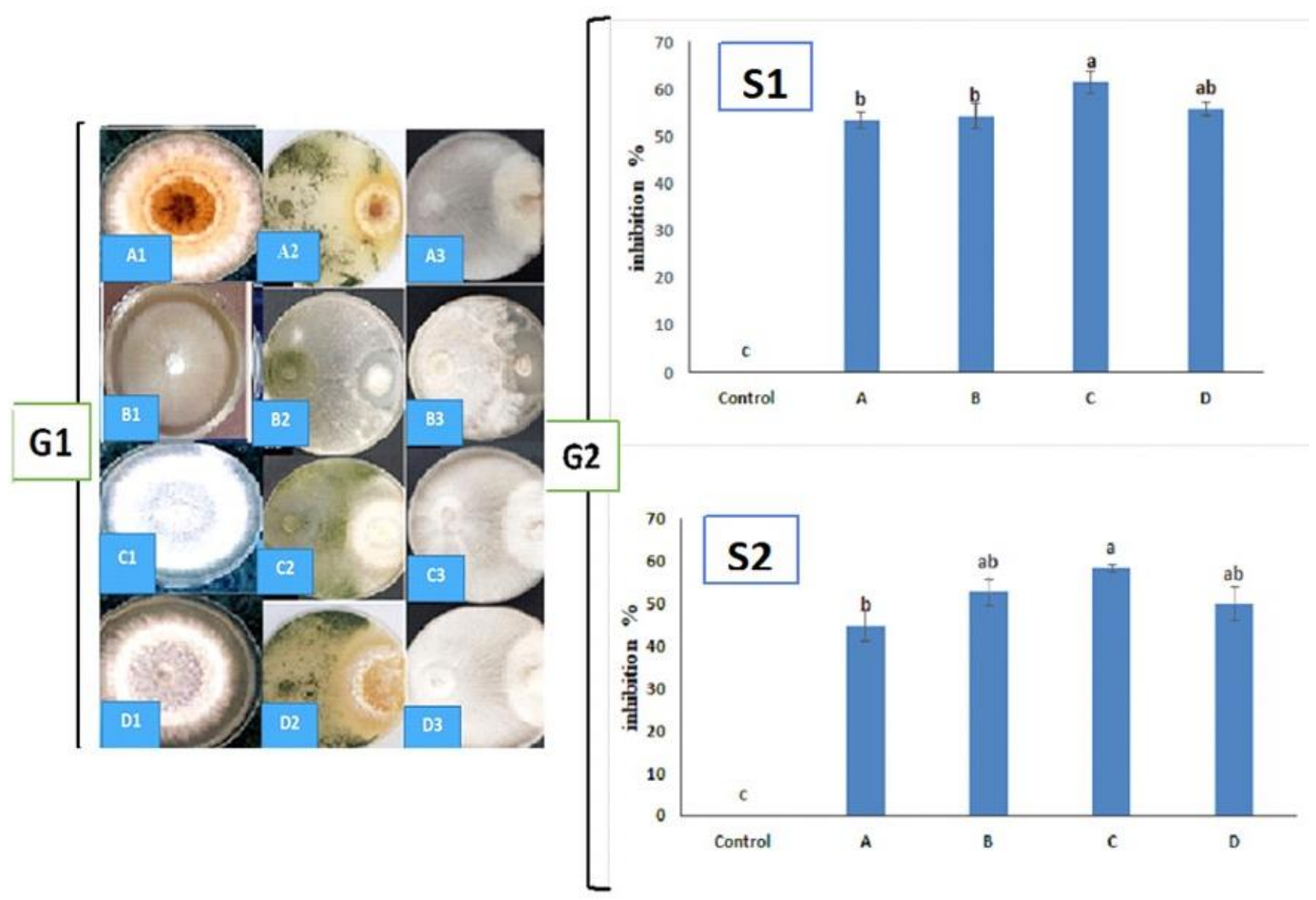

Figure 1. G1: Colony morphology of bioagent fungi (T. harzianum and P. ostreatus) and four strains of Fusarium species. Control treatment (left from A1 to D1): A1: F. incarnatum AJA, B1: F. solani AJA1, C1: F. solani AJA2, and D1: F. oxysporum AJA. Antagonistic activity using a dual plate assay of T. harzianum (middle from A2 to D2) and P. ostreatus (right from A3 to D3) grown on PDA medium (dual culture). G2: The percentage growth inhibition of Fusarium species, F. incarnatum AJA (A), F. solani AJA1 (B), F. solani AJA2 (C), and F. oxysporum AJA (D), under the antagonistic activity of T. harzianum (S1) and P. ostreatus (S2) in dual culture. The data were analyzed by comparison with control after 7 days of growth on PDA at $25 \pm 1^{\circ} \mathrm{C}$. Values are expressed as mean \pm SE of three replicates for each treatment. Similar letters indicate no differences at $\mathrm{P} \leq 0.05$. For the control plate, the pathogenic fungi were individually inoculated at the center of the Petri plate, but they were not treated with the bioagent fungi. 


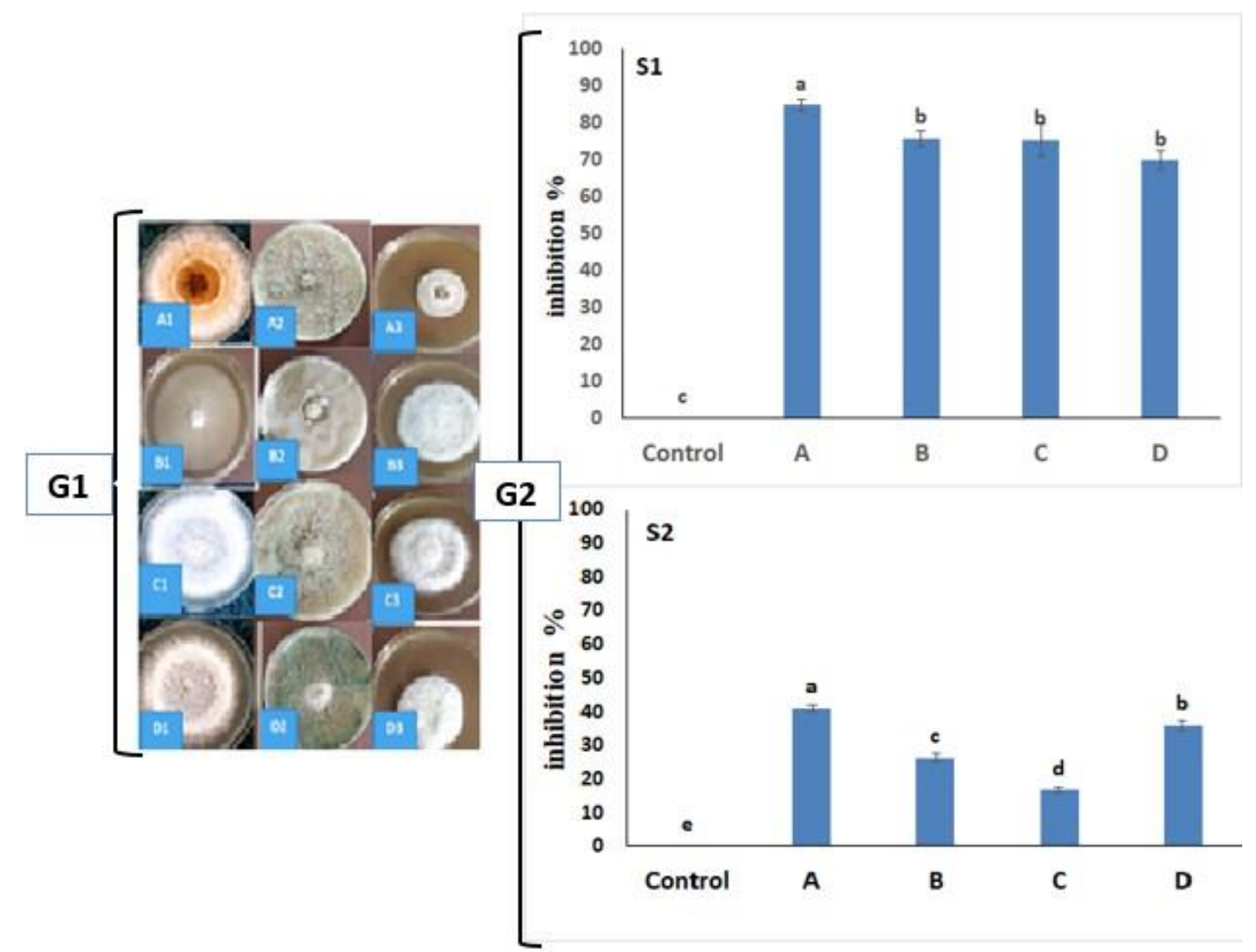

Figure 2. G1: Colony morphology of bioagent fungi (T. harzianum and P. ostreatus) and Fusarium species. Control treatment (left from A1 to D1): A1: F. incarnatum AJA, B1: F. solani AJA1, C1: F. solani AJA2, and D1: F. oxysporum AJA. Antagonistic activity using the fungal filtrate of T. harzianum (middle from A2 to D2) and P. ostreatus (right from A3 to D3) grown on PDA medium (dual culture). G2: The percentage growth inhibition of Fusarium species, F. incarnatum AJA (A), F. solani AJA1 (B), F. solani AJA2 (C), and F. oxysporum AJA (D), treated with filtrates of T. harzianum (S1) and P. ostreatus (S2). The data were analyzed by comparison with the control after 7 days of growth on PDA at $25 \pm 1^{\circ} \mathrm{C}$. Values are expressed as mean \pm SE of three replicates for each treatment. Similar letters indicate no differences at $P \leq 0.05$. For the control plate, the pathogenic fungi were individually inoculated at the center of the Petri plate, but they were not treated with the filtrates of the bioagent fungi. 


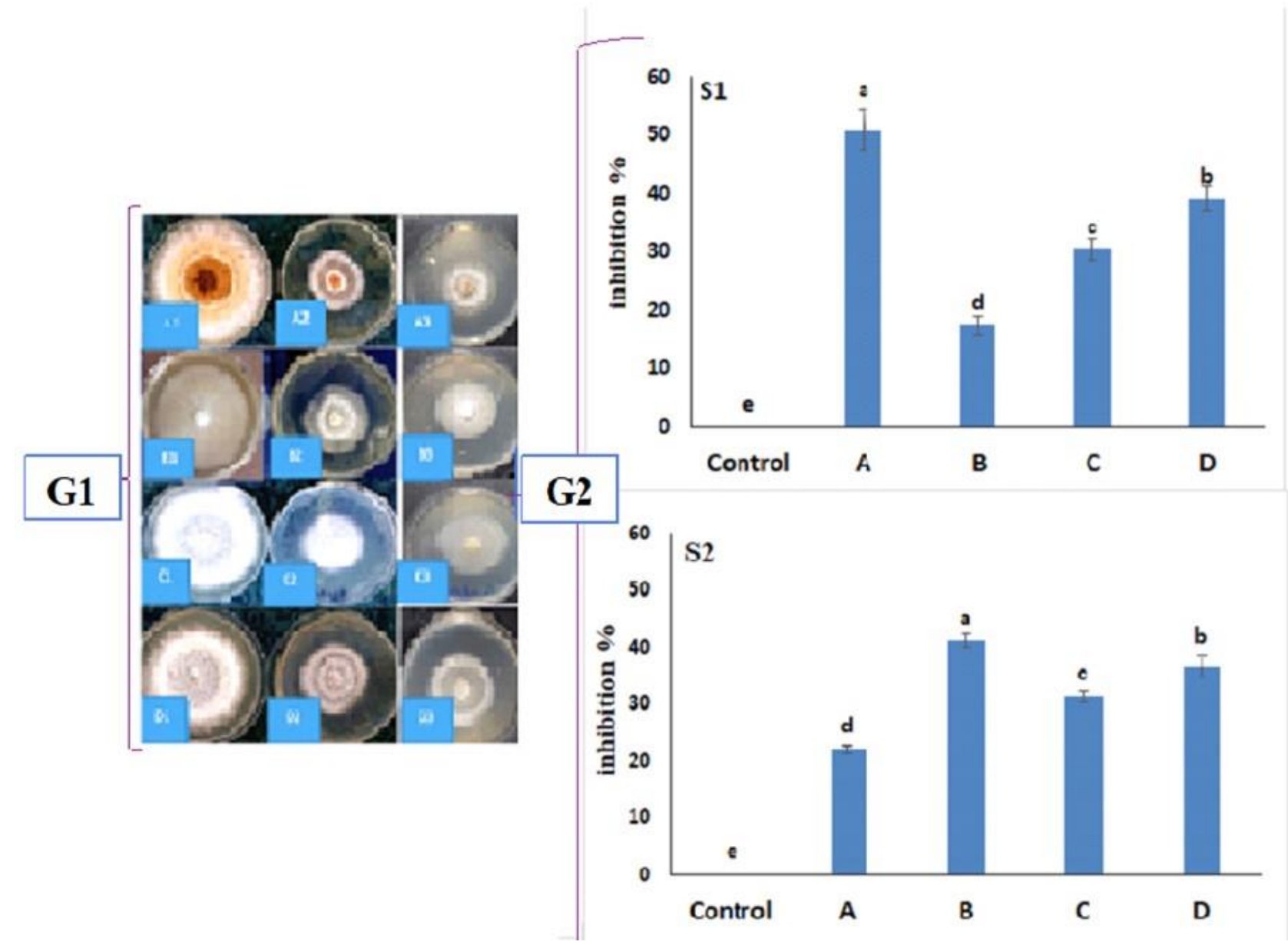

Figure 3. G1: Colony morphology of bioagent fungi (T. harzianum and P. ostreatus) and Fusarium species. Control treatment (left from A1 to D1): A1: F. incarnatum AJA, B1: F. solani AJA1, C1: F. solani AJA2, and D1: F. oxysporum AJA. Antagonistic activity using volatile compounds of T. harzianum (middle from A2 to D2) and P. ostreatus (right from A3 to D3) grown on PDA medium (dual culture). G2: The percentage growth inhibition of Fusarium species, F. incarnatum AJA (A), F. solani AJA1 (B), F. solani AJA2 (C), and F. oxysporum AJA (D), treated with filtrates of T. harzianum (S1) and P. ostreatus (S2). The data were analyzed by comparison with the control after 7 days of growth on PDA at $25 \pm 1^{\circ} \mathrm{C}$. Values are expressed as mean $\pm \mathrm{SE}$ of three replicates for each treatment. Similar letters indicate no differences at $\mathrm{P} \leq 0.05$. For the control plate, the pathogenic fungi were individually inoculated at the center of the Petri plate, but they were not treated with the filtrates of the bioagent fungi.

\section{DISCUSSION}

Several studies have reported the antagonistic activity of Trichoderma species against a variety of fungal phytopathogens (Dennis \& Webster, 1971; Sanjay et al., 2008; Kamala \& Indira, 2011; Abd ElHai et al., 2019). In the present study, the antagonistic activity of $T$. harzianum and $P$. ostreatus was tested in vitro against the pathogenic fungal strains F. incarnatum AJA, F. solani AJA1, F. solani AJA2, and F. oxysporum AJA through the dual culture assay and by assessing the effect of volatile and nonvolatile compounds synthesized by $T$. harzianum and $P$. ostreatus on the growth of these fungal strains.

\section{Antagonistic activity}

The antagonistic fungi $T$. harzianum and $P$. ostreatus caused a substantial reduction in the growth of the pathogenic Fusarium species. The dual plate culture method showed high antagonistic activity of $T$. harzianum and $P$. ostreatus against the four Fusarium strains. The overgrowth of both bioagent fungi over the mycelial growth of the pathogenic fungi was observed, but sporulation 
occurred only for T. barzianum. The colonies of $F$. incarnatum AJA, F. solani AJA1, F. solani AJA2, and $F$. oxysporum AJA treated with $T$. harzianum or $P$. ostreatus were considerably diminished and mostly covered with the mycelia of the bioagent fungi, as both bioagent fungi grew rapidly and could inhibit the growth of the four Fusarium isolates after 7 days of culture. The PGI of the pathogenic fungi ranged from $58.0 \%$ to $62.3 \%$ for $T$. barzianum and from $44.6 \%$ to $58.4 \%$ for P. ostreatus. This finding revealed that the presence of both antagonistic fungi is required for suppressing the growth of pathogenic fungal mycelia (Naglot et al., 2015).

In the current study, both $T$. harzianum and $P$. ostreatus showed interesting antagonistic interactions such as overgrowth and sporulation over the four Fusarium strains. They also exerted a high inhibitory effect and reduced the radial growth of the pathogenic fungi. Because of their ability to produce mucolytic enzymes, both $T$. harzianum and $P$. ostreatus are used as biofertilizers, plant growth promoters, sources for bioremediation, and tools for increasing agricultural productivity (Rahman et al., 2009 Stracquadanio et al., 2020). Moreover, they also have novel potential as biocontrol agents (Hyder et al., 2017).

\section{Nonvolatile compounds}

The fungal filtrate of both T. hariianum and $P$. ostreatus showed a strong inhibitory effect on the fungal pathogens, as the colony growth of all the four Fusarium strains was entirely suppressed by the filtrates of both bioagent fungi. In the control Petri plates, the growth of all Fusarium species covered the entire plate during the same incubation period. The filtrate of $P$. ostreatus greatly affected the morphological characteristics such as the color, texture, and nature of colonies of all Fusarium species. This change in colony characteristics might be due to the diffusion of metabolites synthesized by the bioagent fungi, while pigmentation occurred due to mycelial phenoloxidase or peroxidase activity (Rai et al., 2020).

The PGI varied depending on the type of bioagent fungus and the pathogenic Fusarium strain. T. harzianum induced highest inhibition ranging from $75 \%$ in F. oxysporum AJA to $85 \%$ in F. incarnatum AJA. P. ostreatus also considerably inhibited the growth of Fusarium species, but showed a lesser degree of inhibition than $T$. harzianum; the percentage inhibition ranged from $13.2 \%$ in F. solani AJA1 to $35 \%$ in F. incarnatum AJA ( $\mathrm{P}=0.05)$.

The secondary metabolic products of $P$. ostreatus mycelia are important in preventing the growth of pathogenic fungi (Marques et al., 2018). The increased activity of $P$. salmoneostramineus filtrate is because the chemical composition of the oyster mushroom broth differs depending on the type of fungal product (Adedeji et al., 2016). Pathogenic fungi are inhibited by secondary metabolic products such as polysaccharides, proteins, enzymes, and triterpenoids (Chaudhary \& Tripathi, 2016).

Antibiosis is an antagonistic effect induced by specific or nonspecific microbial metabolites, lytic enzymes, volatile compounds, and other inhibitory substances (Parameswari \& Chinnaswamy, 2011). According to Akyuz \& Kirbag (2009), Trichoderma spp. produce antibiotics such as trichodermin, trichodermol, and herzianolide, which show antagonistic properties against various microorganisms. Ali et al. (2020) observed that the culture filtrate of Bacillus siamensis S3 and Bacillus tequilensis S5 sustained their antifungal activity following thermal treatment by autoclaving and after storage at $4^{\circ} \mathrm{C}$.

\section{Volatile compounds}

Volatile compounds of $T$. harzianum and $P$. ostreatus exhibited different inhibitory effects on Fusarium species. Both these fungal species produced toxic volatile compounds that significantly affected the radial growth of the fungal pathogens but to a varying degree; this was mostly related to the production of volatile organic compounds (VOCs) (Sharma et al., 2013). These secreted metabolites exert an inhibitory effect on various Fusarium species (Küçük \& Kivanç, 2005), and some such metabolites are volatile (Azevedo et al., 2020). In previous investigations, bioagent fungi were found to significantly suppress the growth of F. oxysporum, Rhiroctonia solani, Sclerotium rolfsii, Sclerotinia sclerotiorum, and Alternaria brassicicola (Zeilinger et al., 2016; Meena et al., 2017).

Trichoderma produces a variety of volatile secondary metabolites such as ethylene, hydrogen cyanide, aldehydes, and ketones, which are 
important in plant disease control (Amin et al., 2010; Bhagat et al., 2014). In an earlier study, six isolates of Trichoderma spp. showed potential to produce volatile compounds with activity against seven different fungal plant diseases. Among the six Trichoderma isolates studied, T. viride was found to be the most effective in suppressing the mycelial growth of $F$. oxysporum (41.8\%). The mycelial growth and sclerotia formation in $S$. rolfsii were reduced by $40 \%$ and $48.1 \%$, respectively. Other studies have shown that Trichoderma produces volatile compounds such acetaldehyde, ethylene, acetone, and carbon dioxide (Siddiquee et al., 2014) as well as antibiotics such as trichodermin (Sharma et al., 2016), gliotoxin, viridian (Vargas et al., 2014), and ergokonin (Rai et al., 2016).

Ebadzadsahrai et al. (2020) reported that VOCs of Chromobacterium vaccinii showed the strongest fungal growth inhibition against Phoma sp. and Coleophoma sp. Also, found that the cocultures of bacteria and fungi have emergent volatile metabolome properties.

\section{CONCLUSION}

The antagonistic activity of $T$. harzianum and $P$. ostreatus in reducing the growth of three pathogenic Fusarium species that cause wilt disease in cucumber plants is insufficiently reported in the literature. In the present study, we found that both T. harzianum and P. ostreatus isolates effectively suppressed the growth of Fusarium species. To manage wilt diseases caused by F. incarnatum AJA, F. solani AJA1, F. solani AJA2, and F. oxysporum AJA in cucumber plants, more research on this disease is needed in this field. Although $T$. harzianum and $P$. ostreatus showed differences in their inhibitory effects on Fusarium species, both these species showed effective antagonistic activity and the ability to compete with Fusarium species. To the best of our knowledge, this is the first study to reveal the antagonistic potential of both T. harrianum and P. ostreatus against Fusarium species, and the findings of this study may act as a catalyst for further research.

\section{ACKNOWLEDGEMENTS}

The authors express their gratitude to the Deanship of the College of Sciences and the Head of the Department of Biology at the University of Babylon, Iraq, for providing scientific facilities. The authors would also like to thank Prof. Dr. Majeed M. Dewan, College of Agriculture, University of Kufa, Iraq, for providing Pleurotus ostreatus isolate.

\section{CONFLICT OF INTEREST}

The authors declare that they have no conflicts of interest.

\section{REFERENCES}

Abd El-Hai, K. M., \& Ali, A. A. 2019. Formulation of Trichoderma, Saccharomyces and Rhizobium metabolites against damping-off and root rot pathogens in peanut plant. Asian Journal of Biological Sciences 12: 114-121.

Adedeji, K. O., \& Aduramigba, M. O. 2016. In vitro evaluation of spent mushroom compost on growth of Fusarium oxysporium f. sp. lycopersici. Advances in Plants and Agriculture Research 4(4): 332-339.

Akyuz, M., \& Kirbag, S. 2009. Antimicrobial activity of Pleurotus eryngii var. ferulae grown on various agro-wastes. EurAsian Journal of BioSciences 3(1): 58-63.

Ali, M., Ren, H., Ahmed, T., Luo, J., An, Q., Qi, X., \& Li, B. 2020. Antifungal effects of rhizospheric Bacillus species against bayberry twig blight pathogen Pestalotiopsis versicolor. Agronomy 10 (11): 1811.

Amin, F., Razdan, V. K., Mohiddin, F. A., Bhat, K. A., \& Sheikh, P. A. 2010. Effect of volatile metabolites of Trichoderma species against seven fungal plant pathogens in-vitro. Journal of Phytology 2(10).

Azevedo, D. M., da Silva Rocha, F., Fernandes, M. D., da Costa. C. A., Muniz, M. D., Barroso, P. D., Amaral, F. L., \& do Rosário Barbosa, D. M. 2020. Antagonistic effect of Trichoderma isolates and its metabolites against Fusarium solani and $F$. oxysporum in chickpea. Brazilian Journal of Development 6 (6): 36344-61.

Bhagat, S., Birah, A., Kumar, R., \& Yadav, M. S., Chattopadhyay, C. 2014. Plant disease management: prospects of pesticides of plant origin. In Advances in Plant Biopesticides (pp. 119129). Springer, New Delhi.

Chaudhary, R., \& Tripathi, A. 2016. Interactions between mushrooms and fungi in dual-culture experiments. International Journal of Advanced Research 6: 482-493.

Chen, J. H, Xiang W., Cao, K. X., Lu, X., Yao, S.C., Hung, D., Huang, R. S., \& Li, L B. 2020. Characterization of volatile organic compounds emitted from endophytic Burkholderia cenocepacia ETR-B22 by SPME-GC-MS and their inhibitory activity against various plant fungal pathogens. Molecules 25(17): 3765 . 
Dennis, C., \& Webster, J. 1971. Antagonistic properties of speciesgroups of Trichoderma: I. Production of non-volatile antibiotics. Transactions of the British Mycological Society 57(1): 25-IN3.

Ebadzadsahrai, G., Higgins Keppler, E. A., Soby, S. D., \& Bean, H. D. 2020. Inhibition of fungal growth and induction of a novel volatilome in response to Chromobacterium vaccinii volatile organic compounds. Frontiers in Microbiology.

Fokkema, N. J. 1978. Fungal antagonisms in the phyllosphere. Annals of Applied Biology 89(1): 115-9.

Heydari, A., \& Pessarakli, M. A. 2010 Review on biological control of fungal plant pathogens using microbial antagonists. Journal of Biological Sciences 10(4): 273-290.

Hyder, S., Inam-ul-Haq, M., Bibi, S., Humayun, A, Ghuffar, S., \& Iqbal, S. 2017. Novel potential of Trichoderma spp. as biocontrol agent. Journal of Entomology and Zoology Studies 5(4): 214-222.

Kamala, T., \& Indira, S. 2011. Evaluation of indigenous Trichoderma isolates from Manipur as biocontrol agent against Pythium aphanidermatum on common beans. 3 Biotech 1(4): 217-225.

Khan, R. A., Najeeb, S., Hussain, S., Xie, B., \& Li, Y. 2020. Bioactive secondary metabolites from Trichoderma spp. against phytopathogenic fungi. Microorganisms 8 (6): 817.

Kottb, M., Gigolashvili, T., Großkinsky, D. K., \& Piechulla, B. 2015. Trichoderma volatiles effecting Arabidopsis: from inhibition to protection against phytopathogenic fungi. Frontiers in Microbiology 29 (6): 995.

Küçük, Ç., \& Kivanç, M. 2005. In vitro antifungal activity of strains of Trichoderma harzianum. Turkish Journal of Biology 28(2-4): 111-115.

Kuzmanovska, B., Rusevski, R., Jankulovska, M., \& Oreshkovikj, K. B. 2018. Antagonistic activity of Trichoderma asperellum and Trichoderma harzianum against genetically diverse Botrytis cinerea isolates. Chilean Journal of Agricultural Research 78(3): 391-399.

Li, N., Alfiky, A, Wang W, Islam M, Nourollahi K, Liu X, \& Kang S. 2018. Volatile compound-mediated recognition and inhibition between Trichoderma biocontrol agents and Fusarium oxysporum. Frontiers in Microbiology 31(9): 2614.

Marques, E., Martins I., \& Mello, S. C. 2018. Antifungal potential of crude extracts of Trichoderma spp. Biota Neotropica 22: 18.

Meena, M., Swapnil, P., Zehra, A., Dubey, M. K., \& Upadhyay, R. S. 2017. Antagonistic assessment of Trichoderma spp. by producing volatile and non-volatile compounds against different fungal pathogens. Archives of Phytopathology and Plant Protection 50 (13-14): 629-648.

Mishra, R., \& Sarma, V. V. 2018. Current perspectives of endophytic fungi in sustainable development. In Fungi and their role in sustainable development: Current perspectives (pp. 553-584). Springer, Singapore.

Miyashira, C. H. Tanigushi D. G., Gugliotta A. M., \& Santos D. Y. 2010. Comparison of radial growth rate of the mutualistic fungus of Atta sexdens rubropilosa forel in two culture media. Brazilian Journal of Microbiology 41(2): 506-511.

Naglot, A., Goswami, S., Rahman, I., Shrimali, D. D., Yadav, K. K., Gupta, V. K., Rabha, A. J., Gogoi, H. K., \& Veer, V. 2015. Antagonistic potential of native Trichoderma viride strain against potent tea fungal pathogens in North East India. The Plant Pathology Journal 31(3): 278.

Nasir, A., Al-Janabi, H. J. K., \& Al-Janabi, J. K. A. 2021. Morphological and molecular characterizations of four Fusarium species infecting cucumber in green house. Under press.

Oszako, T., Voitka, D. Stocki, M., Stocka, N., Nowakowska, J. A., Linkiewicz, A., Hsiang, T., Belbahri, L., Berezovska, D., \& Malewski, T. 2021. Trichoderma asperellum efficiently protects Quercus robur leaves against Erysiphe alphitoides. European Journal of Plant Pathology 159: 295-308.
Owaid, M. N., Al-Saeedi, S. S., Abed, I. A., Shahbazi, P., \& Sabaratnam, V. 2017. Antifungal activities of some Pleurotus species (Higher Basidiomycetes). Walailak Journal of Science and Technology (WJST) 14(3): 215-224.

Owaid, M. N., Al-Saeedi, S. S., \& Al-Assaffii I. A. 2015. Antimicrobial activity of mycelia of oyster mushroom species (Pleurotus spp.) and their liquid filtrates (in vitro). Journal of Medical and Bioengineering 4(5).

Parameswari, V., \& Chinnaswamy P. 2011. An in vitro study of the inhibitory effect of Pleurotus florida a higher fungi on human pathogens. Journal of Pharmacy Research 4: 1948194-9.

Rahman, M. A., Begum, M. F., \& Alam, M. F. 2009. Screening of Trichoderma isolates as a biological control agent against Ceratocystis paradoxa causing pineapple disease of sugarcane. Mycobiology 37(4): 277-285.

Rai, N., Limbu, A. K., \& Joshi, A. 2020. Impact of Trichoderma sp. in agriculture: A mini-review. Journal of Biology and Today's World 9(7): 1-5.

Rai, S., Kashyap, P. L., Kumar, S., Srivastava, A. K., \& Ramteke, P. W. 2016. Comparative analysis of microsatellites in five different antagonistic Trichoderma species for diversity assessment. World Journal of Microbiology and Biotechnology 32 (1): 8 .

Rajani, P., Rajasekaran, C., Vasanthakumari, M. M., Olsson, S. B., Ravikanth, G., \& Shaanker, R. U. 2021 Inhibition of plant pathogenic fungi by endophytic Trichoderma spp. through mycoparasitism and volatile organic compounds. Microbiological Research 1(242): 126595.

Sanjay, R., Ponmurugan, P., \& Baby, U. I. 2008. Evaluation of fungicides and biocontrol agents against grey blight disease of tea in the field. Crop Protection 27(3-5): 689-694.

Sharma, A., Diwevidi, V. D., Singh, S., Pawar, K. K., Jerman, M., Singh, L. B., Singh S., \& Srivastawav, D. 2013. Biological control and its important in agriculture. International Journal of Biotechnology and Bioengineering Research 4(3): 175-180.

Sharma, M., Sharma, P., Raja, M., Kumar, K., Chandra, S., \& Sharma, R. 2016. Trichothecene (trichodermin) production in Trichoderma. International Journal of Current Microbiology and Applied Sciences 5(7): 382-386.

Siddiquee, S. 2014. Recent advancements on the role and analysis of volatile compounds (VOCs) from Trichoderma. In Biotechnology and biology of Trichoderma. (pp. 139-175). Elsevier.

Speckbacher, V., Ruzsanyi, V., Wigger, M., \& Zeilinger, S. 2020. The Trichoderma atroviride strains P1 and IMI 206040 differ in their light-response and VOC production. Molecules 25(1): 208.

Stracquadanio, C., Quiles, J. M., Meca, G., \& Cacciola, S. O. 2020. Antifungal activity of bioactive metabolites produced by Trichoderma asperellum and Trichoderma atroviride in liquid medium. Journal of Fungi 6(4): 263.

Tamur, H. A., Mohsin, L. Y., Al-Janabi, J. K., \& Zahraa, A. N. 2018. Marasmiellus palmivorus as a new causal agent of reed wilt disease in Iraq. Pakistan Journal of Biotechnology 15(1): 2531.

Vargas, W. A., Mukherjee, P. K., Laughlin, D., Wiest, A., MoranDiez, M. E., \& Kenerley, C. M. 2014. Role of gliotoxin in the symbiotic and pathogenic interactions of Trichoderma virens. Microbiology 160(10): 2319-2330.

Zeilinger, S., Gruber, S., Bansal, R., \& Mukherjee, P. K. 2016. Secondary metabolism in Trichoderma-chemistry meets genomics. Fungal Biology Reviews 30(2): 74-90. 